\title{
PERFIL GESTACIONAL, OBSTÉTRICO E NEONATAL NA UNIDADE DE TERAPIA INTENSIVA DE UM HOSPITAL PÚBLICO DO INTERIOR DA BAHIA
}

\author{
GESTACIONAL, OBSTETRIC AND NEONATAL PROFILE IN THE INTENSIVE \\ CARE UNIT IN A PUBLIC HOSPITAL OF THE INTERIOR OF BAHIA
}

\author{
Felix Meira Tavares, Hellen Tamara Ferraz Mathias Lima Silva
}

Faculdade Independente do Norte

\begin{abstract}
The objective of the study was to trace the gestational and obstetric maternal profile and to identify the characteristics of neonates hospitalized in an Neonatal Intensive and Semi Intensive Care Unit (NICU). Methods: This is a descriptive study with a cross-sectional design and a quantitative approach. The data collection was done through documentary data in medical records and through questionnaires, which the informants had as their informants. The sample consisted of 18 newborns and 11 mothers, of which $72,73 \%$ were from other cities. Regarding the gestational maternal profile: $63,64 \%$ were between 20 and 25 years old, single and selfdeclared with brown color; 81,82\% had only one child and 45,45\% presented C1 in the economic classification. Regarding the obstetric profile: $61,11 \%$ of the newborns were born with a surgical delivery, 67\% were Preterm and with birth weight ranging from 867,69 $\pm 2275,83$ grams. Most newborns presented some level of Pulmonary Injury. Conclusion: the studied population has a profile that compose the need for neonatal hospitalization in the NICU, such as low socioeconomic status, abdominal delivery and prematurity. The mortality risk of neonates admitted to the NICU was 10\%, and all of them had Acute Lung Injury.
\end{abstract}

Key words: Unidades de Terapia Intensiva Neonatal, Cuidado Pré-Natal, Assistência Perinatal.

\section{Resumo}

O estudo teve como objetivos traçar o perfil materno gestacional e obstétrico e apontar as características dos neonatos internados em uma Unidade de Terapia Intensiva e semi intensiva (UTIN). Métodos: Trata-se de um estudo descritivo com delineamento transversal $e$ abordagem quantitativa. A coleta de dados foi realizada através de dados documentais em prontuários médicos e por meio de questionários os quais tiveram como informantes as genitoras. A amostra foi composta por 18 neonatos e 11 genitoras das quais $72,73 \%$ eram provenientes de outras cidades. Quanto ao perfil materno gestacional: 63,64\% possuíam entre 20 a 25 anos, solteiras e se auto-declararam com cor parda; $81,82 \%$ possuíam apenas um filho $e$ 45,45\% apresentaram classe C1 na classificação econômica. Quanto ao perfil obstétrico: $61,11 \%$ dos neonatos nasceram de parto cirúrgico, $67 \%$ era Pré-termo e com peso ao nascer com variação entre $867,69 \pm 2275,83$ gramas. A maior parte dos neonatos apresentou algum nível de Lesão Pulmonar. Conclusão: a população estudada possui perfil que compõe a necessidade da internação dos neonatos na UTIN, como o baixo nível socioeconômico, parto abdominal $e$ prematuridade. $O$ risco de mortalidade dos neonatos internados na UTIN foi de $10 \%$ sendo que todos eles apresentavam Lesão Pulmonar Aguda.

Palavras chave: Unidades de Terapia Intensiva Neonatal, Cuidado Pré-Natal, Assistência Perinatal. 
Introdução

A permanência de neonatos em Unidade de Terapia Intensiva Neonatal (UTIN) nos primeiros 28 dias está relacionada basicamente à atenção pré, peri e pós-natal, que se não realizadas ou realizadas de maneira insatisfatória poderão ocasionar nascimentos de crianças prematuras e de baixo peso ${ }^{1}$.

O pré-natal deve ser iniciado imediatamente após a descoberta da gravidez, de forma universal e periódica integrando ações preventivas e curativas com no mínimo seis consultas $^{1}$. A assistência pré-natal é importante para a relação mãe-filho.

Observa-se que no Brasil a cobertura da assistência pré-natal é baixa, embora tenha sofrido desdobramentos nos últimos anos, indiferenças nesse tipo de assistência ainda prosseguem. Essa indiferença pode variar segundo as regiões geográficas, sendo a região Nordeste e principalmente o norte do Nordeste há um grande número de mulheres que não comparece ao acompanhamento do pré-natal nas Unidades Básicas de Saúde da Família ${ }^{3}$.

O período perinatal é uma fase onde se deve acompanhar qualidade de saúde da mulher e do neonato, uma vez que muitas ações podem ser desenvolvidas nesse período com objetivo de evitar complicações durante a gestação e após o parto ${ }^{4}$. Segundo a Organização Mundial de Saúde (2004), analisa a fase perinatal como o percurso que se inicia na 22a semana de gestação ou o peso fetal igual ou superior a $500 \mathrm{~g}$, até o 70 dia de vida após o nascimento.

A situação socioeconômica desempenha importante papel no processo saúde-doença, pois existe forte dependência entre o ambiente no qual a criança vive e o seu desenvolvimento físico e psíquico ${ }^{5}$. Cardoso ${ }^{6}$ afirma que o estudo socioeconômico é essencial para identificar fatores que podem influenciar a susceptibilidade geral à doença, e na avaliação dos padrões temporais e espaciais da saúde, doença e bem estar das populações humanas.

O perfil socioeconômico das mães e as características dos neonatos internados em UTIN podem auxiliar os profissionais de saúde a suspeitar quais indivíduos estão mais propensos a tal condição. Desse modo, o presente estudo tem como objetivo traçar um perfil gestacional, obstétrico e neonatal na UTIN e semi intensiva do município de Vitória da Conquista.
Metodologia

Trata-se de um estudo descritivo, com delineamento transversal e enfoque quantitativo. Para caracterização da amostra, foram escolhidas 11 genitoras de neonatos internados na UTIN e Unidade de Terapia Semi Intensiva do Hospital Municipal Esaú Matos, referência para nascimento de neonatos no município de Vitória da Conquista e microrregiões, sendo coletados os dados de 18 prontuários de neonatos internados neste hospital na primeira semana de Julho de 2014, caracterizando assim uma amostragem não probabilística estabelecida por conveniência.

Foram incluídos neste estudo neonatos internados na UTIN e semi intensiva e suas genitoras. A coleta de dados foi iniciada após aprovação pelo Comitê de Ética em Pesquisa da Faculdade Independente do Nordeste sob número de protocolo 26616414.0.0000.5578, respeitando os preceitos éticos da resolução 466/2012 do Conselho Nacional de Saúde.

A aplicação dos instrumentos de coleta foi realizada pelo pesquisador do estudo após assinatura voluntária dos participantes do TCLE. Todas as orientações, bem como os objetivos e procedimentos da pesquisa, riscos, benefícios foram detalhadamente explicadas pelo pesquisador.

Os instrumentos consistiram em dois questionários aplicados às mães e um escore realizado com os dados coletados nos prontuários dos neonatos. $\mathrm{O}$ primeiro questionário consistiu das seguintes perguntas: idade, estado civil, renda familiar e partos anteriores, referentes aos dados maternos; e tipo de parto, idade gestacional e peso ao nascer, referentes aos dados obstétricos.

O segundo questionário utilizado foi o de classificação econômica do Brasil validado pela Associação Brasileira de Empresas de Pesquisas $(A B E P)^{7}$. Este instrumento tem como função estimar o poder de compra das famílias urbanas abandonando a ambição de classificar a população em termos de classe sociais, classifica os itens de compra, grau de instrução e se há presença de empregadas mensalista. Utiliza-se de sistema de pontos que são acumulados e calculados criando um parâmetro confiável.

Este critério atribui pontos em função de cada característica domiciliar. É feito então uma correspondência entre as faixas de pontuação e os estratos de classificação econômica definidas por A1, A2, B1, B2, C1, C2, D e E. 
O último instrumento foi o escore SNAP II (Score for Neonatal Acute Phisiology), proposto por Richardson e colaboradores ${ }^{8}$, este instrumento contem seis variáveis fisiológicas, incluindo a Pressão Arterial Média em milímetros de mercúrio ( $\mathrm{mmHg}$ ), temperatura em Celsius $\left({ }^{\circ} \mathrm{C}\right)$, relação entre a pressão arterial parcial de oxigênio e a fração inspirada de oxigênio $\left(\mathrm{PaO}_{2} / \mathrm{FiO}_{2}\right)$, potencial Hidrogeniônico $(\mathrm{pH})$, Diurese em mililitros por quilograma por hora $(\mathrm{ml} / \mathrm{kg} / \mathrm{h})$, Convulsões múltiplas, Peso ao nascer em gramas (g), PIG (pequeno para idade gestacional) e Escala de Apgar pontuando valores fisiológicos. O escore varia de 0 (baixa severidade) a 115 (alta severidade) A finalidade deste instrumento é avaliar a gravidade e o nível de morbidade e mortalidade dos neonatos internado em UTIN e semi intensiva.

Inicialmente foi realizada a análise dos prontuários para preenchimento do escore SNAP Il de todos os neonatos incluídos no estudo. Após esta fase, os demais questionários sobre a classificação econômica, dados maternos e obstétricos foi aplicado com as genitoras que compareceram na UTIN no período de coleta, não sendo autorizada pelo Hospital a coleta de dados das mães que ainda estavam internadas no mesmo.

Os dados obtidos foram armazenados e processados utilizando o Programa Microsoft Excel 2010 e descritos em Média \pm DesvioPadrão. $\mathrm{O}$ valor de $(\mathrm{N})$ corresponde ao número de genitoras e neonatos que participaram da pesquisa. Os resultados foram descritos com cálculo das frequências simples e ocorrências para os dados referentes ao perfil materno e obstétrico das genitoras e pontuação da Classificação Econômica e SNAP II.

\section{Resultados}

Observando os dados obstétricos, todas as mães realizaram pré-natal com 6,82 \pm 1,27 consultas. O tipo de parto mais freqüente foi o abdominal (58,82\%). A maioria das crianças nasceram prematuras $(70,59 \%)$ sendo a média da idade gestacional de $35,39 \pm 4,15$ semanas. A média de peso ao nascer dos neonatos foi de $2275,83 \pm 867,69$ gramas, sendo que apenas $27,78 \%$ apresentaram baixo peso ao nascimento.

A média da idade das participantes foi de $23,6 \pm 6,1$ anos, a maioria delas (72,73\%) residiam em cidades vizinhas a Vitória da Conquista e apenas $27,27 \%$ residiam na mesma. A cor auto declarada mais prevalente foi a Parda com $63,64 \%$ dos relatos. Levando em consideração a estrutura familiar, a maioria das genitoras eram solteiras $(63,64 \%)$ e primíperas (81,82\%).

Em relação à escolaridade, as genitoras tinham em sua maioria o ensino médio completo $(54,55 \%)$ e a maioria relatou possuir nenhuma renda (45,45\%). Nesse sentido, na Classificação Econômica as classes B2 e C1 foram as mais frequentes, cada uma delas com 36,36\% das entrevistadas. As características descritivas da amostra: faixa etária, cidade, estado civil, classificação econômica, cor (auto declarada), número de filhos podem ser visualizadas na Tabela 1.

Das 18 crianças internadas na UTIN e na Unidade de Terapia semi intensiva, apenas 9 possuíam dados hemogasométricos nos prontuários, podendo dessa forma realizar o cálculo do escore SNAP II para avaliar a gravidade e a morbidade e mortalidade dos RNs internados. A maioria das crianças apresentou o escore de 12 a 23 (valor preditivo de mortalidade de 10\%), correspondendo a $66,7 \%$ dos casos (Figura 1).

$\mathrm{Na}$ avaliação desta escala foi observado que todas os neonatos tiveram algum grau de lesão pulmonar, uma vez que a média da relação $\mathrm{PaO}_{2} / \mathrm{FiO}_{2}$ foi de 223,61 \pm 91,23. A Pressão Arterial Média (PAM) mostrou-se em 65,56 \pm $18,53 \mathrm{mmHg}$. A escala de Apgar pontuou no primeiro minuto $6,11 \pm 2,26$ e no quinto minuto $8,00 \pm 1,50$. Estes dados pedem ser observados na Tabela 3.

Tabela 1. Dados Obstétricos das crianças internadas na UTIN.

\begin{tabular}{lcc}
\hline Dados obstétricos & $\mathbf{N}$ & $\%$ \\
\hline Tipo de parto & 7 & $38,89 \%$ \\
Vaginal & 11 & $61,11 \%$ \\
Abdominal & & \\
Idade gestacional & 12 & $66,67 \%$ \\
29 à 37 semanas & 6 & $33,33 \%$ \\
38 a 42 semanas & & \\
Peso ao nascer & 10 & $55,56 \%$ \\
Abaixo de 2 Kg & 8 & $44,44 \%$ \\
2 a 3 Kg & & \\
\hline
\end{tabular}


Tabela 2. Características sociodemográficas e econômicos das genitoras das crianças internadas na UTIN.

\begin{tabular}{lcc}
\hline \multicolumn{1}{c}{ Dados sociodemográficos e econômicos } & $\mathbf{N}$ & $\%$ \\
\hline Faixa etária & 7 & $63,64 \%$ \\
15 à 25 anos & 4 & $36,36 \%$ \\
26 à 35 anos & 3 & $27,27 \%$ \\
Cidade & 8 & $72,73 \%$ \\
Vitória da Conquista & & \\
Outras cidades & 4 & $36,46 \%$ \\
Estado civil & & $63,64 \%$ \\
Casada & 7 & \\
Solteira & & $36,36 \%$ \\
Classificação econômica & 4 & $45,45 \%$ \\
B2 & 5 & $9,09 \%$ \\
C1 & 1 & $9,09 \%$ \\
C2 & 1 & \\
D & & $18,18 \%$ \\
COR (auto declarada) & 2 & $9,09 \%$ \\
Branca & 1 & $63,64 \%$ \\
Negra & 7 & $9,09 \%$ \\
Parda & 1 & \\
Amarela & & $81,82 \%$ \\
Número de filhos & & $18,18 \%$ \\
Um filho & 9 & 2 \\
Dois filhos & & \\
\hline
\end{tabular}

Tabela 3. Dados de monitorização dos neonatos internadas na UTIN.

\begin{tabular}{|c|c|}
\hline Dados de monitorização & Média $\pm d p$ \\
\hline Pressão Arterial Média (mmhg) & $65,56 \pm 18,53$ \\
\hline Temperatura $\left({ }^{\circ} \mathrm{C}\right)$ & $35,81 \pm 0,51$ \\
\hline Relação $\mathrm{paO}_{2} / \mathrm{fio}_{2}$ & $223,61 \pm 91,23$ \\
\hline Ph sérico & $7,36 \pm 0,08$ \\
\hline Diurese (ml em 24h) & $30,49 \pm 19,64$ \\
\hline Apgar 1을 minuto & $6,11 \pm 2,26$ \\
\hline Apgar 50 minuto & $8,00 \pm 1,50$ \\
\hline
\end{tabular}

Figura 1: Escore SNAP II dos neonatos internados na UTIN, apontando os valores dos escores preditivos de mortalidade. Até $11(3 \%) ; 12$ a $23(10 \%) ; 24$ a $32(53 \%)$ e 33 a $50(83 \%)^{9}$.

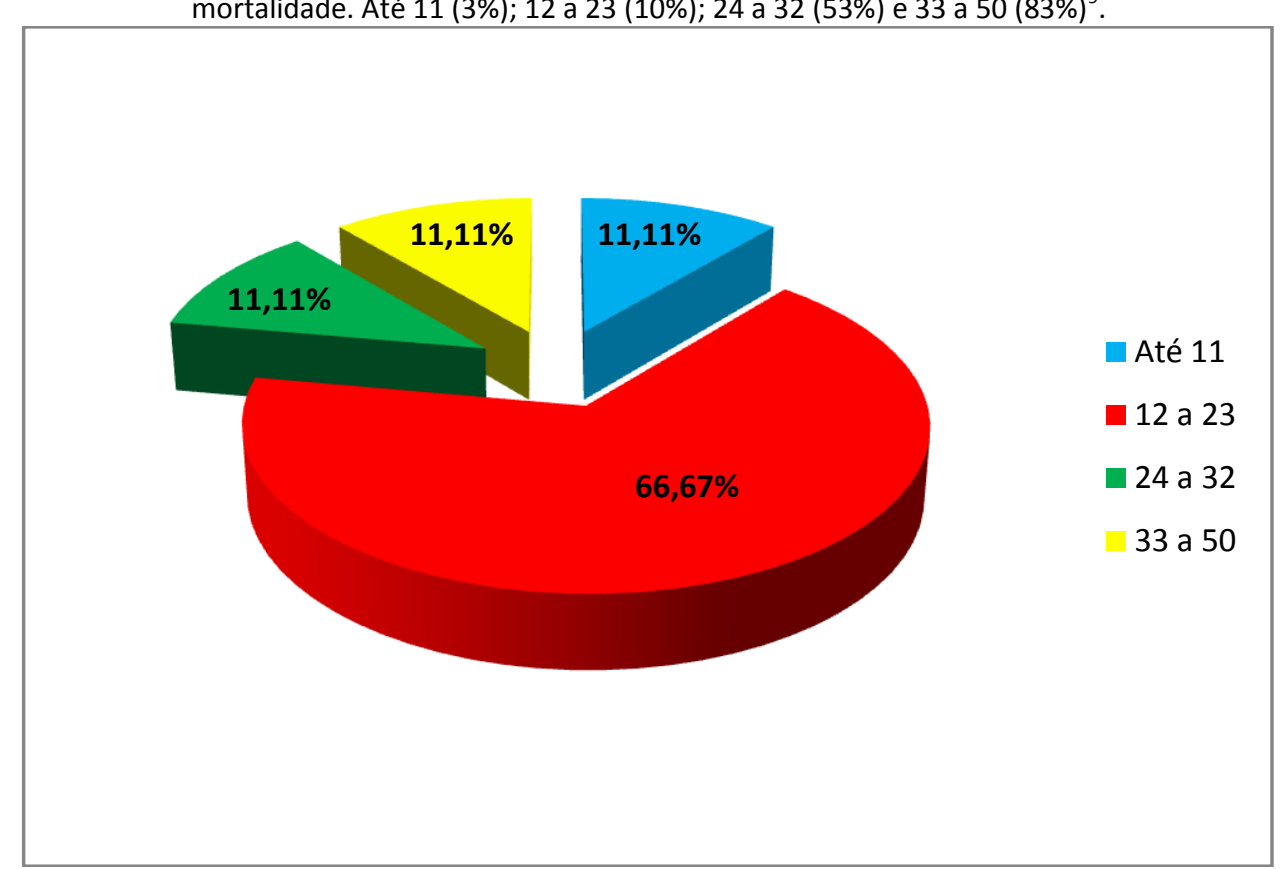

PERFIL GESTACIONAL, OBSTÉTRICO E NEONATAL NA UNIDADE DE TERAPIA INTENSIVA DE UM HOSPITAL PÚBLICO DO INTERIOR DA 


\section{Discussão}

Foi observado que a maioria das mães tem uma idade inferior a 25 anos $(63,64 \%)$, estudos apontam que quanto menor a idade da gestante, mais tempo será despendido para buscar um serviço de pré-natal $^{10}$. No entanto, outros estudos apontam que as condições de vida e saúde da genitora, incluindo o comparecimento em consultas obstétricas, são mais importantes do que a idade das mesmas ${ }^{10,11}$.

Os resultados da assistência pré-natal estão relacionados ao contentamento da gestante aos serviços prestados, fazendo com que as mesmas tenham maior adesão às consultas ${ }^{12}$. A média de consultas pré-natais encontradas no estudo foi de $6,82 \pm 1,27$, um pouco abaixo do preconizado pelo Ministério da Saúde, que recomenda mais de sete consultas $^{13}$. Tal fato pode interferir o acompanhamento gestacional através do diagnóstico precoce de condições mórbidas, bem como no período perinatal, complicações puerperais e até mesmo a mortalidade ${ }^{14}$.

A renda familiar quantifica a qualidade e os hábitos de vida das famílias relacionadas à educação, alimentação, saúde e habitação ${ }^{5}$. Neste estudo observamos que a maioria das mães entrevistadas apresentavam baixa renda, sendo classificadas segundo o critério brasileiro de classificação econômica na classe $C_{1}$, que possui renda até $\mathrm{R} \$ 1.685$ mensais. Segundo Zambonato et al. $^{15}$ a condição socioeconômica está intimamente ligado ao risco de nascimentos de crianças pequenas para a idade gestacional e propensas a disfunções orgânicas, juntamente a fatores de risco gestacionais como a préeclâmpsia.

O hospital onde foi realizada a coleta de dados é referência regional na área maternoinfantil. Das mães entrevistadas, $72,73 \%$ eram oriundas de outros municípios, demonstra a necessidades de implementação de novo hospitais que atenda esse público no sudoeste da Bahia.

Resultados semelhantes ao desta pesquisa também foram encontrados no estudo de Barbosa et al. ${ }^{16}$, que ao avaliar o perfil dos neonatos com desconforto respiratório internados em um hospital público do interior da Bahia concluiu que maioria de genitoras possuíam baixo nível socioeconômico, sendo procedentes de outros municípios da região e com um número de consultas pré-natais abaixo do preconizado.
Com relação aos dados obstétricos, 66,67\% dos neonatos apresentaram idade gestacional entre 29 e 37 semanas. Sabe-se que maior parte da população de uma Unidade de terapia intensiva é composta de pré termos, os neonatos que nascem antes das 37 semanas de gestação tem maior probabilidade de complicações nos sistemas pulmonar, neurológico e sensorial, podendo comprometer o desenvolvimento psicomotor e comportamental $^{17}$.

A pontuação do escore de gravidade dos neonatos (SNAP II), um preditor de sobrevida neonatal, foi de 12 a 23 em $66,67 \%$ dos casos, que demonstra um risco de mortalidade de $10 \%$. Tal gravidade foi observada pelos parâmetros de monitorização encontrados nos neonatos. Um exemplo é baixa relação $\mathrm{PaO}_{2} / \mathrm{FiO}_{2}$ encontrada, com media de 223,61 $\pm 91,23$. A relação entre oxigenação e necessidade de oxigenioterapia suplementar maior que 200 e menor que 300 é um indicativo de Lesão Pulmonar Aguda ${ }^{18}$.

A escala de Apgar é uma avaliação clínica do recém-nascido que analisa os sistemas cardiorrespiratório e neuromuscular. É uma ferramenta útil para identificar as crianças que necessitam de cuidados adicionais, quando aplicada com 1 minuto de vida e, novamente, com 5 minutos $^{19}$. Um escore de Apgar de 7-10 denota uma criança sadia, que possivelmente não terá problemas futuros. Quando $<7$, é sinal de alerta para uma atenção maior ${ }^{19}$.

A média da escala de Apgar foi de 6,11 \pm 2,26 no primeiro minuto e $8,00 \pm 1,50$ no quinto minuto, demonstrando certo grau de recuperação. Os fatores de risco para índice de Apgar baixo no primeiro minuto incluíram vários agravantes, entre estes idade materna, fatores de ordem social, história obstétrica, assistência pré-natal, como os também encontrados no estudo de Costa, A. L., et. al. ${ }^{20}$ que avaliou os Fatores de risco materno associados à necessidade de unidade de terapia intensiva neonatal.

\section{Conclusão}

A população estudada possui algumas características que compõe a necessidade da internação dos neonatos na UTIN e na semi intensiva. Tais características envolvem o baixo nível socioeconômico, parto abdominal e prematuridade. $\mathrm{O}$ risco de mortalidade dos neonatos internados na UTIN foi de $10 \%$ sendo que todos eles apresentavam Lesão Pulmonar Aguda. 
Este estudo considera a necessidade de novas pesquisas, com maior número de genitoras entrevistadas e neonatos internados para a obtenção de resultados mais expressivos. No entanto, os achados no presente estudo apontam para a necessidade de programas de intervenção elaborados por equipes multidisciplinares, de modo a dar uma melhor assistência famílias de baixa renda, visando dar orientações sobre a importância do acompanhamento pré-natal e dos cuidados durante a gestação com a finalidade de evitar a internação do neonato nas UTIN.

\section{Referências}

1. Federação Brasileira Das Sociedades De Ginecologia E Obstetrícia. Assistência pré-natal. Manual de orientação. 11 de junho de 2006. (FEBRASGO, Projeto Diretrizes: Associação Médica Brasileira e Conselho Nacional de Medicina).

2. Cunha MA, Mamede MV, Dotto LMG, Mamede F. Assistência Pré-Natal: Competências Essenciais Desempenhadas por Enfermeiros. Esc Anna Nery Rev Enferm 2009 jan-mar; 13 (1): 000.(http://dx.doi.org/10.1590/S1414$81452009000100020)$.

3. Ministério da Saúde; Saúde reprodutiva: gravidez, assistências ré-natal, parto e baixo peso ao nascer. Saúde Brasil 2004, uma análise da situação de saúde. 1 ed. Brasília 2004. p. 69 -84.

4. Costa, R. F, Queiroz, M. V. O, Brasil, E. G. M, Marques, J. F, Xavier, E. O. Assistência à mulher na fase perinatal: opinião de profissionais da saúde. Revista de enfermagem UFPE. Recife, V.7 N.5, p.4505 -13, jun., 2013.

5. Carvalho,J. A, Bezerra, A. L. T, Freitas, A. R. R, Pereira, J. V. N. M, Oliveira, J. L. T. M. Avaliação do nível socioeconômico e hábitos familiares de educando de escolas públicas municipais de João Pessoa/ PB. XII Encontro de extensão, 2010

6. Cardoso, H.F.V, A quantificação do estudo socioeconômico em populações contemporâneas e históricas: dificuldades, algumas orientações e importância na investigação orientada para saúde. Antropologia portuguesa. v. 22/23,: p.247-272, 2005/2006.

7. Associação Brasileira de Empresas de Pesquisa [homepage on the Internet].

8. Critério de classificação econômica Brasil. [cited 2012 Sept 14].

9. Richardson DK, Phibbs CS, Gray JE,
McCormick MC, Workman- Daniels K, Goldmann DA. Birth weight and illness severity: independent predictors of neonatal mortality. Pediatrics 1993; 91:969-75. (https://www.ncbi.nlm.nih.gov/pubmed/847481 8).

10. Silveira $\mathrm{RC}$ et al. Valor preditivo dos escores de SNAP e SNAP-PE na mortalidade neonatal. Jornal de Pediatria - v. 77, n 6, 2001. (http://dx.doi.org/10.1590/S0021-

\section{7).}

11. Ximenes FMA et al. A influência da idade materna sobre as condições perinatais. RBPS 2004; $\quad 17 \quad$ (2): $\quad 56-60$. (http://www.redalyc.org/html/408/40817103/).

12. Azevedo et al. Efeito da Idade Materna sobre os Resultados Perinatais. RBGO - v. 24, n 3, $2002 . \quad$ (http://dx.doi.org/10.1590/S010072032002000300006).

13. Silveira, D. S.; Santos, I. S. \& Costa, J. S. D. Atenção pré-natal na rede básica: uma avaliação da estrutura e do processo. Caderno de saúde pública. Rio de Janeiro, v.17 n.1 p.131-139, jan-fev, 2001. (http://dx.doi.org/10.1590/S0102311X2001000100013).

14. Ministério da Saúde. Pré-natal e Puerpério: atenção qualificada e humanizada manual técnico. Brasília: Mistério da Saúde; 2006 15. Silva CF, Leite AJM, Almeida NMGS. Fatores de risco para mortalidade infantil em município do Nordeste do Brasil: linkage entre bancos de dados de nascidos vivos e óbitos infantis -2000 a 2002. Rev. bras. epidemiol. 2006; 9(1): $69-80$

16. Zambonato AMK, Pinheiro RT, Horta BL, Tomasi E. Fatores de risco para nascimento de crianças pequenas para idade gestacional. Rev Saúde Pública. 2004; 38(1): 24-29. (http://dx.doi.org/10.1590/S0034$89102004000100004)$.

17. Barbosa VA et al. Perfil dos neonatos com desconforto respiratório internados em um hospital público do interior da Bahia. Rev.Saúde.Com 2013; 9(1): 24-32. ( http://www.uesb.br/revista/rsc/ojs/index.php/rs c/article/view/285).

18. Palha, M. Recém-nascidos com fatores de riscos no período Peri-natal. Centro de desenvolvimento infantil diferenças. (www.diferencas.net)

19. AMIB Associação de Medicina Intensiva Brasileira. Recomendações para o Reconhecimento e Abordagem do Recémnascido, da Criança e do Adolescente com 
Doença Grave causada pelo vírus Influenza A H1N1. São Paulo, 2009.

20. Oliveira, TG et al. Escore de Apgar e mortalidade neonatal em um hospital localizado na zona sul do município de São Paulo. Einstein. 2012;10(1):22-8.

(http://dx.doi.org/10.1590/S167945082012000100006).

21. Costa, AL. Fatores de risco materno associados à necessidade de unidade de terapia intensiva neonatal Rev Bras Ginecol Obstet. 2014; 36(1):29-34.

(http://dx.doi.org/10.1590/s010072032007000900010).

\section{Endereço para Correspondência}

Faculdade Independente do Nordeste

e-mail: felixmeira@gmail.com

Recebido em 13/01/2017

Aprovado em 25/05/2017

Publicado em 03/08/2017 